\title{
Toward improved statistical methods for analyzing Cotinine-Biomarker health association data
}

Tulay Koru-Sengul ${ }^{1,2^{*}+}$, John D Clark ${ }^{1,3+}$, Lora E Fleming ${ }^{1,4}$ and David J Lee

\begin{abstract}
Background: Serum cotinine, a metabolite of nicotine, is frequently used in research as a biomarker of recent tobacco smoke exposure. Historically, secondhand smoke (SHS) research uses suboptimal statistical methods due to censored serum cotinine values, meaning a measurement below the limit of detection (LOD).

Methods: We compared commonly used methods for analyzing censored serum cotinine data using parametric and non-parametric techniques employing data from the 1999-2004 National Health and Nutrition Examination Surveys (NHANES). To illustrate the differences in associations obtained by various analytic methods, we compared parameter estimates for the association between cotinine and the inflammatory marker homocysteine using complete case analysis, single and multiple imputation, "reverse" Kaplan-Meier, and logistic regression models.

Results: Parameter estimates and statistical significance varied according to the statistical method used with censored serum cotinine values. Single imputation of censored values with either $0, L O D$ or $L O D / \sqrt{ } 2$ yielded similar estimates and significance; multiple imputation method yielded smaller estimates than the other methods and without statistical significance. Multiple regression modelling using the "reverse" Kaplan-Meier method yielded statistically significant estimates that were larger than those from parametric methods.

Conclusions: Analyses of serum cotinine data with values below the LOD require special attention. "Reverse" Kaplan-Meier was the only method inherently able to deal with censored data with multiple LODs, and may be the most accurate since it avoids data manipulation needed for use with other commonly used statistical methods. Additional research is needed into the identification of optimal statistical methods for analysis of SHS biomarkers subject to a LOD.
\end{abstract}

\section{Background}

A biomarker is a laboratory measure of a biological process [1]. The lowest quantity of a biomarker that can be distinguished from the lack of that biomarker is the biomarker's limit of detection (LOD), below which the level of biomarker cannot be accurately measured. One important yet unresolved issue in analyzing biomarker data arises when biomarker measurements fall below the LOD (i.e. "non-detects", "left-censored").

Statistical analyses of data that include biomarker measurements below the LOD are complicated since

\footnotetext{
* Correspondence: tsengul@med.miami.edu

+ Contributed equally

'Department of Epidemiology and Public Health at Leonard Miller School of Medicine, University of Miami, Miami, Florida, USA

Full list of author information is available at the end of the article
}

precise quantitative levels cannot always be determined [1]. In all analyses involving biomarkers with a LOD, researchers working with biomarker data inevitably have to deal with data containing non-detects, and must decide how to combine non-detects with values above the LOD for analysis. The choice of an appropriate strategy for dealing with data affected by LODs requires an understanding of both experimental and statistical procedures. Until now, the common practice has been to impute (i.e. substitute a single value, such as a half of the detection limit, for each measurement below the LOD), and to then conduct the analysis under the assumption that the imputed values are the actual observed values [2-4]. This assumption may be invalid, leading to biased results, especially when trying to predict small exposure-health outcome associations $[5,6]$.

\section{Ciomed Central}


Analytic issues with secondhand smoke (SHS) exposure biomarkers (e.g. cotinine, 4-(methylnitrosamino)1-(3-pyridyl)-1-butanol (NNAL) [NNAL]) arise due to a large percentage of measurements below the LOD. Serum cotinine, a metabolite of nicotine, is widely used in research as an objective measure of recent tobacco smoke exposure. The use of traditional statistical methods to analyze serum cotinine measurements often introduces bias into the study results due to measurements falling below the LOD, potentially affecting the accuracy of analytic results and validity of study conclusions [1-4]. Furthermore, this bias may be greatest in studies investigating the health effects of tobacco smoke exposures at very low exposure levels, a situation where the subjects' cotinine levels are more likely to be undetectable. Therefore, the impact of low-level SHS exposure on human disease may be underestimated using traditional statistical methods for handling biomarkers below the LOD $[1,2]$.

The purpose of our research was to demonstrate and compare the performances of commonly used statistical methods as a case study for analyzing associations between serum cotinine measurements with non-detects and levels of the inflammatory marker homocysteine using data from the 1999-2004 National Health and Nutrition Examination Surveys (NHANES) [7].

\section{Methods}

This project's data consisted of adults 18 and older participating in the 1999-2004 NHANES, a cross-sectional study designed to assess the health and nutritional status of adults and children annually in the United States $(\mathrm{n}=31,126)$. The continuous NHANES survey combines interviews and physical examinations; the resulting data can be pooled across multiple years. Anonymous survey data and related documents were obtained from the NHANES website http://www.cdc.gov/nchs/nhanes. htm.

Subjects included in this analysis were non-smoking adults age 20 years and older as defined by a serum cotinine level less than or equal to $3.08 \mathrm{ng} / \mathrm{mL}$ or by self report of not smoking within the past 5 days. This particular cotinine threshold for adults has reportedly a sensitivity of $96.3 \%$ and a specificity of $97.4 \%$ for differentiating smokers from non-smokers [8]. The subjects included in this analysis had detectable or undetectable serum cotinine levels and complete information on additional variables of interest (i.e. homocysteine, age, gender, race/ethnicity, and SHS exposure) in order to focus on the issue of properly handling left-censored serum cotinine data $(n=9,488)$.

In the NHANES surveys, serum cotinine was assessed using an isotope dilution high performance liquid chromatography atmospheric pressure chemical ionization tandem mass spectrometric method [9]. The laboratory test detection limit for serum cotinine in NHANES was $0.05 \mathrm{ng} / \mathrm{mL}$ for 1999-2000 and part of 2001-2002. Improvements in the laboratory testing methods reduced the LOD to $0.015 \mathrm{ng} / \mathrm{mL}$; this lower detection limit was used for the 2001-2002 and the 2003-2004 NHANES. For the $1999-2000$ cycle, $37.7 \%$ of values were below the LOD $(0.05 \mathrm{ng} / \mathrm{mL})$, while $22.6 \%$ were below the LOD for the 2001-2002 cycle (either 0.015 $\mathrm{ng} / \mathrm{mL}$ or $0.05 \mathrm{ng} / \mathrm{mL}$ ) and $16.9 \%$ were below the LOD for the 2003-2004 cycle $(0.015 \mathrm{ng} / \mathrm{mL})$. Plasma homocysteine was analyzed using an automated fluorescence polarization immunoassay [9].

We compared commonly used statistical methods (Table 1) as a case study for analyzing serum cotinine with measurement values below the LOD using parametric and non-parametric techniques: complete case analysis, single and multiple imputation methods, "reverse" Kaplan-Meier method, and logistic regression models [2].

\section{Methods of Handling Left-Censored Data}

Complete-case analysis is a method of analysis that incorporates only subjects with serum cotinine values above the LOD. This is a widely used method due to its simplicity, but is highly inefficient since it reduces sample size and produces bias and loss of precision in the estimation [10].

Imputation is the practice of replacing undetectable serum cotinine with "plausible" values [11]. After imputation, data can be analyzed as if imputed values were actual observed values. In single imputation, each nondetect value is replaced with an estimate, but this does not account for the sampling variability produced by imputed values. Single imputation generally results in the underestimation of variance, which directly affects confidence intervals and statistical tests. In our analysis, we performed single imputation analyses substituting one of three different commonly used substitution values for non-detect cotinine measurements: 0 , LOD, and LOD $/ \sqrt{ } 2$. Depending on the LOD of the cotinine measurement method, single imputation values changed when the LOD changed across survey cycles (i.e. $0.05 \mathrm{ng} /$ $\mathrm{mL}$ for $1999-2000$ and part of 2001-2002; $0.015 \mathrm{ng} / \mathrm{mL}$ for part of 2001-2002 and 2003-2004).

Multiple imputation is a simulation-based approach which can provide a good solution to missing data problems; and it has been used extensively with complex national surveys [12-16]. The basic idea of multiple imputation is to replace each non-detect with a vector of more than two plausible values from the predictive data distribution [13-15]. In general, multiple imputation results in unbiased estimates, uses all available data, preserves both sample size and statistical power, and 
Table 1 Characteristics and Comparisons of the Statistical Methods Used for Analysis of Biomarker Data with Limits of Detection

\begin{tabular}{|c|c|c|c|c|c|}
\hline Method & $\begin{array}{l}\text { n used } \\
(\% \text { total) }\end{array}$ & $\mathrm{n}$ below LOD & $\begin{array}{l}\text { Transforms censored data } \\
\text { to categorical data }\end{array}$ & $\begin{array}{l}\text { Replaces censored values } \\
\text { with imputed value }\end{array}$ & $\begin{array}{l}\text { Inherently able to deal } \\
\text { with multiple LOD? }\end{array}$ \\
\hline Complete Case & $5,865(62)$ & 0 & $\mathrm{No}^{*}$ & $\mathrm{No}^{*}$ & $\mathrm{No}^{*}$ \\
\hline Single Imputation & $9,488(100)$ & 3,623 & No & Yes & No \\
\hline Multiple Imputation & $9,488(100)$ & 3,623 & No & Yes & Yes \\
\hline Logistic regression & $9,488(100)$ & 3,623 & Yes & No & Yes \\
\hline "Reverse" Kaplan-Meier & $9,488(100)$ & 3,623 & No & No & Yes \\
\hline
\end{tabular}

LOD: limit of detection

*Excludes censored data

reflects the sampling variability. After imputation, any statistical software designed for analyzing complete data can be used.

In our multiple imputation method, we considered non-detect cotinine measurements as missing values based on an imputation model derived from multivariate normal distribution used to construct the predictive distribution for non-detected serum cotinine that included: homocysteine, serum cotinine, age, gender (female/male), race/ethnicity (Non-Hispanic White/Other), and selfreported SHS exposure (yes/no). The number of imputations was set to 10 (i.e. 10 complete datasets including both the detected and imputed non-detected serum cotinine measurements in addition to other variables of interest such as homocysteine, age, gender, race/ethnicity, and self-reported SHS exposure). Ten completed datasets are usually sufficient for multiple imputation [14].

Two different multiple imputation models were used for creating the imputed serum cotinine with LOD. They were both based on a multivariate normal distribution which takes into account the correlation between the variables included in the imputation model. The first model included only homocysteine and serum cotinine; the second imputation model not only included homocysteine and serum cotinine, but also included age, gender, race/ethnicity, and self-reported SHS exposure to take into account the strength of the relationship among all other variables. The imputation model predicted distribution for non-detected serum cotinine from the subjects' homocysteine, age, gender (female/ male), race/ethnicity (Non-Hispanic White/Other), and self-reported SHS exposure (yes/no).

The "reverse" Kaplan-Meier method is a non-parametric method that does not require a probabilistic distribution to estimate the survival function from time-to-event type of biological data [17]. It is frequently used with right-censored survival data. For left-censored data, an equivalent estimator can be obtained by Turnbull estimator, which is equivalent to the "reverse" Kaplan-Meier estimator. We performed the "reverse" Kaplan-Meier method by considering left-censored serum cotinine as a "time-to-event" outcome.
Logistic regression is a modelling technique for dichotomous outcomes [18]. We fitted a logistic regression model using dichotomized serum cotinine (below vs. above LOD) as the outcome variable, and the continuous homocysteine values as a predictor variable.

In all of the methods, the univariate regression models for serum cotinine included only continuous homocysteine values as a predictor. The multiple regression models not only included continuous homocysteine values, but also age, gender (female/male), race/ethnicity (Non-Hispanic White/Other), and self-reported SHS exposure (yes/no).

\section{Statistical analysis}

To illustrate the differences in the estimates obtained among different analytical methods for handling LOD, study variables were regressed on the inflammatory marker homocysteine. The NHANES survey weights were not incorporated in any of these analyses to show the differences between the methods as a case study under a random sampling schema. In this case study, the primary objective was to compare the parameter estimates from univariate and multiple regression models that quantify the relationship between inflammatory marker homocysteine and serum cotinine subject to LOD. The univariate regression models for inflammatory marker homocysteine included only serum cotinine as a predictor variable; the multiple regression models included additional variables (as described above). The regression coefficient estimate of serum cotinine, its standard error, and the ratio of the regression coefficient to its standard error as well as the statistical significance were reported and compared among all the methods. All of the analyses were implemented using SAS version 9.2 for Windows statistical software (SAS Institute Inc., Cary, NC, USA). Statistical significance was attained with p-value $<0.05$.

\section{Results}

Table 1 summarizes and compares the characteristics of the different methods considered for analyses of leftcensored serum cotinine data. Except for the complete 
case analysis method, all the methods preserved the sample size. The methods differed on how censored values were treated in the analyses. The complete case analysis method was not suitable since it simply excluded all of the censored cotinine values from the analysis. Logistic regression models were also not suitable methods since it collapsed cotinine values into a dichotomous categorical variable (detectable vs. undetectable).

Methods that replaced censored measurements with imputed values were the single and multiple imputation methods. This happens for both single and multiple imputation when either: 1) a non-detect is substituted with an imputed value; or 2) when more than one LOD exists and all values below the highest LOD are treated as non-detects. The methods inherently able to deal with multiple LOD were the multiple imputation, "reverse" Kaplan-Meier method, and logistic regression. Based on the three criteria listed in table 1, the "reverse" Kaplan-Meier method was the most efficient method because it did not require transformation of data to a categorical variable, did not replace censored data with an imputed value, and was able to handle data with multiple LODs.

Parameter estimates and statistical significance for serum cotinine varied according to different methods (Table 2). All the methods found positive associations between serum cotinine and homocysteine in both univariate and multiple regression models. In all methods of analyses, univariate regression estimates (range: 0.001 - 0.524) were smaller than multiple regression estimates (range: 0.020 - 1.093). The multiple imputation method reported the smallest estimates in both the univariate and multiple regression models, while the logistic regression models resulted in the largest regression estimates. The only method that did not reveal statistically significant associations between serum cotinine measurements and levels of homocysteine was multiple imputation.

\section{Discussion}

The results of this analysis suggest that different statistical methods for handling LODs can result in variable parameter estimates with resulting $\mathrm{p}$-values both above and below the level of $5 \%$ when investigating the associations between serum cotinine and the inflammatory marker homocysteine. While all methods revealed positive associations between serum cotinine and homocysteine, the multiple imputation method did not yield significant parameter estimates. The complete case analysis, single imputation, "reverse" Kaplan-Meier, and logistic regression demonstrated statistically significant positive associations between cotinine and homocysteine levels.

Significant variation between methods was seen in the size of the estimates using both univariate and multiple regression models; a 524 and 55 fold difference was seen in the size of estimates between the multiple imputation and logistic regression using univariate and multiple regression models, respectively. The smallest ratio (estimate/SE) was observed with multiple imputation, while the largest in the logistic regression model. Single imputation using different values for substitution also resulted in slight variation in the size of both univariate and multiple regression models; regression estimates increased from 0.053 to 0.055 and from 0.079 to 0.083

Table 2 Association between Homocysteine and Serum Cotinine in Non-Smokers: Results by Different Analytical Methods for Analyzing Left-Censored Biomarker Data

\begin{tabular}{|c|c|c|c|c|c|c|c|}
\hline \multirow{3}{*}{$\begin{array}{l}\text { Total } \mathrm{n}=9,488 \\
\text { Method }\end{array}$} & \multirow{3}{*}{ \#subjects included } & \multicolumn{6}{|c|}{ Regression Models } \\
\hline & & \multicolumn{3}{|c|}{ Univariate* } & \multicolumn{3}{|c|}{ Multiple* } \\
\hline & & Estimate (SE) & Estimate/SE & p-value & Estimate (SE) & Estimate/SE & p-value \\
\hline Complete Case & 5,865 & $0.027(0.011)$ & 2.455 & 0.0137 & $0.053(0.009)$ & 5.889 & $<0.0001$ \\
\hline \multicolumn{8}{|l|}{ Single Imputation with } \\
\hline 0 & 9,488 & $0.055(0.011)$ & 5.000 & $<0.0001$ & $0.083(0.008)$ & 10.375 & $<0.0001$ \\
\hline LOD & 9,488 & $0.053(0.011)$ & 4.818 & $<0.0001$ & $0.079(0.009)$ & 8.778 & $<0.0001$ \\
\hline LOD/sqrt(2) & 9,488 & $0.054(0.011)$ & 4.909 & $<0.0001$ & $0.081(0.009)$ & 9.000 & $<0.0001$ \\
\hline Multiple Imputation & 9,488 & $0.001(0.025)$ & 0.040 & 0.9787 & $0.020(0.026)$ & 0.769 & 0.4367 \\
\hline Logistic Regression*** & 9,488 & $0.524(0.055)$ & 9.527 & $<0.0001$ & $1.093(0.076)$ & 14.382 & $<0.0001$ \\
\hline "Reverse" Kaplan-Meier *** & 9,488 & $0.012(0.015)$ & 0.800 & $<0.0001$ & $0.222(0.017)$ & 13.059 & $<0.0001$ \\
\hline
\end{tabular}

LOD: limit of detection; sqrt: squared root

Estimate (SE): regression coefficient and its standard error between Serum Cotinine and Homocysteine

*Univariate regression models for inflammatory marker Homocysteine include serum cotinine only as a covariate; Type-I error rate $=5 \%$.

**Multiple regression models for inflammatory marker Homocysteine include serum cotinine, age in years, gender (female/male), race/ethnicity (non-Hispanic

White/Others), and second hand smoking status (yes/no) as covariates; Type-I error rate $=5 \%$.

*** Outcome of these methods is left-censored serum cotinine. 
for univariate and multiple regression models, respectively, as the imputation value was changed from the LOD to 0 .

Since the true regression coefficient parameter is not known, we do not know for sure which result is the most accurate. Multiple imputation, although believed to be appropriate for complex sample survey data and endorsed in the past for use with NHANES data, is subject to many of the same limitations as complete case and single imputation analysis due to the replacement of censored values with values that were not measured directly [19]. While the creation of binary (below vs. above LOD) or ordinal (below LOD, above LOD as low, medium, high) categorical data from censored data does not violate any assumptions of logistic regression, such statistical methods have much less power compared to "reverse" Kaplan-Meier due to aggregating biomarker measurements into subgroups. Additionally, the grouping and subsequent loss of statistical power with the creation of categorical/ordinal cotinine variables may not be able to detect small associations, such as with the health effects of very low levels of SHS exposure. The "reverse" Kaplan-Meier method, however, appears to be both the most efficient and the most accurate method for this analysis since it is inherently able to handle censored data with multiple LODs, and it avoids the need for data manipulation (needed with imputation methods or logistic regression).

\section{Conclusions}

Researchers who use tobacco smoke exposure biomarkers, such as serum cotinine, should be aware that commonly used analytic techniques for handling leftcensored data may bias study results by using suboptimal statistical methods, especially in studies investigating the health effects of SHS exposure due to typically small effect sizes. Analysis of left-censored data requires special attention, as different methods may yield different results as shown in our case study. Many commonly used statistical methods do not properly handle left-censored serum cotinine data because these methods either exclude subjects with undetectable cotinine levels or impute values that are treated as actual observed values. Our analysis suggests "reverse" Kaplan-Meier is the preferred method of analysis of serum cotinine with censored data and with multiple LODs until more in depth research on optimal methods of analysis for left-censored data finds its place in the scientific literature.

Statistical simulation studies are needed to compare the results and to generalize the conclusions to other surveys, as well as to other left-censored biomarker data. In statistical simulation studies, one can compare the estimates obtained from different methods with the true estimates to understand bias and error introduced by varying the different scenarios (such as the percentage of LOD, the magnitude of the correlation between the left-censored biomarker, and the other variables of interest).

Considering the frequent use of SHS biomarkers in public health research and their impact on public health policy, research is needed into the most appropriate statistical methods for use with SHS biomarkers, and welldefined guidelines need to be developed for analyzing SHS biomarkers data.

\section{Acknowledgements}

This work was supported by the Flight Attendant Medical Research Institute (FAMRI) Clinical Innovator Awards to Dr. Koru-Sengul and Dr. Lee, the National Institute of Environmental Health Sciences (NIH F30 ES015969), the National Institute for Occupational Safety and Health grants (R01 OH003915), and the European Union Convergence funding (ECEHH, PCMD, University of Exeter). The content is solely the responsibility of the authors and does not necessarily represent the official views of any of the Institutes.

\section{Author details}

${ }^{1}$ Department of Epidemiology and Public Health at Leonard Miller School of Medicine, University of Miami, Miami, Florida, USA. ${ }^{2}$ Sylvester Comprehensive Cancer Center at Leonard Miller School of Medicine, University of Miami, Miami, Florida, USA. ${ }^{3}$ Department of Internal Medicine, Kaiser Permanente, Los Angeles, California, USA. ${ }^{4}$ European Centre of Environment and Human Health (ECEHH), Peninsula College of Medicine and Dentistry, Truro, Cornwall, UK.

\section{Authors' contributions}

TKS and JDC contributed equally to the manuscript. TKS managed the data, performed all statistical analysis and wrote the manuscript. JDC managed the data and wrote the manuscript. LEF and DJL assisted in the writing of the manuscript. All authors read and approved the final manuscript version.

\section{Competing interests}

The authors declare that they have no competing interests.

Received: 18 March 2011 Accepted: 3 October 2011

Published: 3 October 2011

\section{References}

1. Schisterman EF, Little RJ: Opening the black box of biomarker measurement error. Epidemiology 2010, 21(Suppl 4):S1-S3.

2. Helsel D: Nondetects and Data Analysis: Statistics for Censored Environmental Data. John Wiley \& Sons; 2005.

3. Helsel DR: Fabricating data: How substituting values for nondetects can ruin results, and what can be done about it. Chemosphere 2006, , 65: 2434-2439.

4. Krishnamoorthy $\mathrm{K}$, Mallick A, Mathew T: Model based imputation approach for data analysis in the presence of nondetectable values: Normal and Related Distributions. Annals of Occupational Hygiene 2009, 59: 249-261.

5. Armstrong BG: Effect of measurement error on epidemiological studies of environmental and occupational exposures. Occupational and Environmental Medicine 1998, , 55: 651-656.

6. Rothman KJ: Methodologic frontiers in environmental epidemiology. Environmental Health Perspectives 1993, 101(Suppl 4):19-21.

7. Clark JD, Wilkinson JD, LeBlanc WG, Dietz NA, Arheart KL, Fleming LE, Lee DJ: Inflammatory markers and secondhand tobacco smoke exposure among U.S. workers. American Journal of Industrial Medicine 2008, , 51: 626-632.

8. Benowitz NL, Bernert JT, Caraballo RS, Holiday DB, Wang J: Optimal serum cotinine levels for distinguishing cigarette smokers and nonsmokers within different racial/ethnic groups in the United States between 1999 and 2004. American Journal of Epidemiology 2009, , 169: 236-248. 
9. NHANES, Laboratory Procedures Manual. 2001 [http://www.cdc.gov/nchs/ data/nhanes/nhanes_01_02/106_b_doc.pdf].

10. Little RJ, Rubin DB: Statistical Analysis with Missing Data. Wiley Series in Probability and Statistics., Second 2002.

11. Donders AR, van der Heijden GJ, Stijnen T, Moons KG: Review: a gentle introduction to imputation of missing values. Journal of Clinical Epidemiology 2006, , 59: 1087-1091.

12. Barnard J, Meng XL: Applications of multiple imputation in medical studies: from AIDS to NHANES. Statistical Methods in Medical Research 1999, 8: 17-36.

13. Harel O, Zhou XH: Multiple imputation: review of theory, implementation and software. Statistics in Medicine 2007, , 26: 3057-3077.

14. Rubin D: Multiple Imputation For Nonresponse In Surveys. John Wiley \& Sons Inc; 1987.

15. Schafer JL: Analysis of Incomplete Multivariate Data. CRC Monographs on Statistics \& Applied Probability. 1997.

16. Schafer J: Analyzing the NHANES III Multiply Imputed Data Set: Methods and examples. 2001 [http://www.nber.org/nhanes/nhanesll//ftp.cdc.gov/ pub/Health_Statistics/NCHS/Datasets/NHANES/NHANESIII/7a/doc/analyzing. pdf].

17. Gillespie BW, Chen Q, Reichert H, Franzblau A, Hedgeman E, Lepkowski J, Adriaens P, Demond A, Luksemburg W, Garabrant DH: Estimating Population Distributions When Some Data Are Below a Limit of Detection by Using a Reverse Kaplan-Meier Estimator. Epidemiology 2010, 21: S64-S70

18. Albert PS, Harel O, Perkins N, Browne R: Use of Multiple Assays Subject to Detection Limits With Regression Modeling in Assessing the Relationship Between Exposure and Outcome. Epidemiology 2010, , 21: S35-S43.

19. The Third National Health and Nutrition Examination Survey, (NHANES III, 1988-1994). Multiply Imputed Data Set (Series 11, No. 7A). [ftp://ftp. cdc.gov/pub/Health_Statistics/NCHS/nhanes/nhanes3/7A/readme.txt], Accessed 7/7/11.

doi:10.1186/1617-9625-9-11

Cite this article as: Koru-Sengul et al:: Toward improved statistical methods for analyzing Cotinine-Biomarker health association data. Tobacco Induced Diseases 2011 9:11.

\section{Submit your next manuscript to BioMed Central and take full advantage of:}

- Convenient online submission

- Thorough peer review

- No space constraints or color figure charges

- Immediate publication on acceptance

- Inclusion in PubMed, CAS, Scopus and Google Scholar

- Research which is freely available for redistribution

Submit your manuscript at www.biomedcentral.com/submit
Biomed Central 